\title{
Cardiac cysticercosis: A rare cause of myocardial infarction
}

Tiago Bernardo Nery, MD, Cláudio Leo Gelape, PhD, Luiz Guilherme Passaglia, MA, and Gabriel Assis Lopes do Carmo, PhD, Belo Horizonte, Minas Gerais, Brazil

\author{
From the Cardiac Surgery and Cardiology Division, Hospital das Clínicas da Universidade Federal de Minas \\ Gerais (HC-UFMG), Belo Horizonte, Minas Gerais, Brazil. \\ Disclosures: Authors have nothing to disclose with regard to commercial support. \\ Received for publication Aug 3, 2017; revisions received Sept 23, 2017; accepted for publication Oct 12, 2017; \\ available ahead of print Nov 20, 2017. \\ Address for reprints: Tiago Bernardo Nery, MD, 1811, Oscar Freire St, Apt 102, Pinheiros, São Paulo, Brazil \\ 05409-011 (E-mail: tiago.bernardo@hc.fm.usp.br). \\ J Thorac Cardiovasc Surg 2018; $155: \mathrm{e} 155-8$ \\ $0022-5223 / \$ 36.00$ \\ Copyright (c) 2017 by The American Association for Thoracic Surgery \\ https://doi.org/10.1016/j.jtcvs.2017.10.058
}

Video clip is available online.

Cysticercosis is the infection by the larval stage of Taenia solium. This disease can affect multiple human organs, most commonly the central nervous system, subcutaneous tissue, and muscles. When the myocardium is involved, the patient is usually free of symptoms, but we report a case of cardiac cysticercosis as a cause of myocardial infarction.

\section{CLINICAL SUMMARY}

A 59-year-old man came to the emergency department with typical angina 6 hours in duration. He had no previous medical history and reported having stopped smoking 30 years before. The initial electrocardiogram showed a new 1-mm ST-segment elevation of the inferior and lateral walls, and the cardiac troponin level was higher than normal limits. In accordance with the protocol for acute coronary syndrome, the patient was submitted to a coronary angiogram, which showed a distal occlusion in the left anterior descending coronary artery (Figure 1, A, and Videos 1 and 2). An angioplasty was not feasible, and the patient was stable and free of pain with clinical treatment.

After the investigation, an echocardiogram showed an akinesia of the apical region, with preserved ejection fraction of the left ventricle. The echocardiogram also showed a cystic lesion in the septoapical region without communication with the ventricular cavity (Figure 1, B). The hypothesis of pseudoaneurysm of the left ventricle was considered.

Cardiac magnetic resonance imaging demonstrated an intramural lesion with well-defined borders at the apex of the left ventricle measuring $2.2 \times 3.6 \times 2.0 \mathrm{~cm}$. It extended through the free wall into the pericardium space, and the content corresponded to blood on magnetic resonance imaging. These characteristics suggested a ventricular

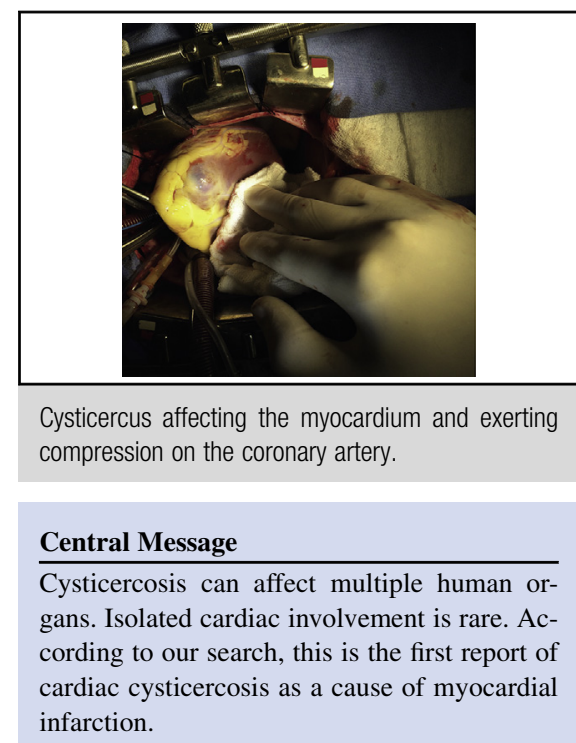

See Editorial Commentary page e159.

pseudoaneurysm (Figure 1, $C$ and $D$ ). The patient underwent surgery because of the high risk of a pseudoaneurysm after acute left ventricular infarction.

The intraoperative finding was an extracoronary cystic lesion $2.5 \mathrm{~cm}$ in size on the diaphragmatic aspect of the apical region in the left ventricle, which was exerting external compression on left anterior descending coronary artery (Figure 2, $A, B$, and $C$ ). The lesion was a semitransparent, thin-walled cyst with mixed content, composed of a serous fluid and a flat, elongated brown structure $15 \mathrm{~mm}$ in size (Figure 2,D). The cyst was opened and enucleated, and the contents were preserved. The lesion was eventually corrected as is conventionally done for a ventricular aneurysm, incorporating polytetrafluoroethylene felt strips. The distal portion of left anterior descending coronary artery at the apex of the heart was sacrificed.

The postoperative period was uneventful, and the patient was discharged on postoperative day 6 . The definitive diagnosis of myocardial cysticercosis was established by the histologic study (Figure 2,E). The patient was symptom free at follow-up, and the team decided not to prescribe specific treatment for cysticercosis.

\section{DISCUSSION}

Cysticercosis is an infection by the larval stage of the parasite $T$ solium, most prevalent in Southern Africa, China, India, Central America, Chile, Brazil, Papua New Guinea, 


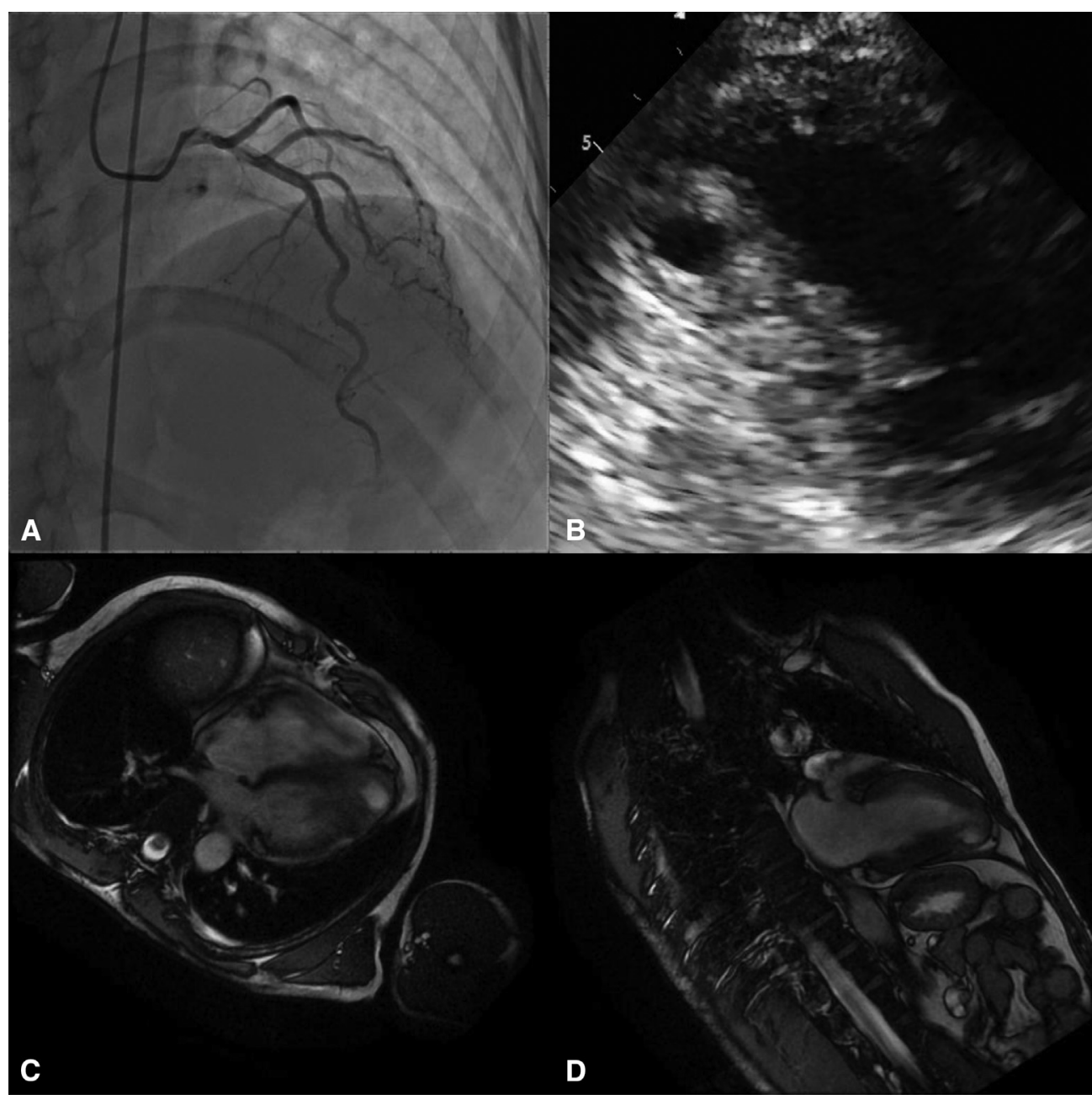

FIGURE 1. A, Coronary angiography demonstrated a distal occlusion of the left anterior descending coronary artery. B, The image of the lesion on echocardiography. $\mathrm{C}$ and D, Cardiac magnetic resonance imaging demonstrated a lesion in the left ventricular apex exerting external compression on the left anterior descending coronary artery.

and non-Islamic South East Asia. ${ }^{1}$ The most affected organs are the central nervous system, subcutaneous tissue, muscle, abdominal organs, and eyes. The prevalence of cardiac involvement in patients with disseminated cysticercosis can be as high as $27 \%$, and the clinical manifestation depends on factors such as the number, location, and stage of the cysticerci and the immune status and comorbid conditions of the host. ${ }^{2-5}$ Cardiac cysticercosis as a cause of myocardial infarction is extremely rare; according to our search, this is the first report.

\section{References}

1. Groom ZC, Protopapas AD, Zochios V. Tropical diseases of the myocardium: a review. Int J Gen Med. 2017;10:101-11.

2. Lino Júnior Rde S, Ribeiro PM, Antonelli EJ, Faleiros AC, Terra SA, dos Reis MA, et al. Developmental characteristics of Cysticercus cellulosae in the human brain and heart [in Portuguese]. Rev Soc Bras Med Trop. 2002;35:617-22.

3. Spina R, Sandaradura I, Puranik R, Lee AS. Cardiac cysticercosis. Int J Cardiol. 2013;168:557-9.

4. Melo RM, Melo Neto AV, Corrêa LC, Melo Filho AV. Restrictive cardiomyopathy due to myocardial cysticercosis [in Portuguese]. Arq Bras Cardiol. 2005;85.

5. Lino RS Jr, Reis MA, Teixeira VP. Occurrence of encephalic and cardiac cysticercosis (Cysticercus cellulosae) in necropsy [in Portuguese]. Rev Saude Publica. 1999;33:495-8. 


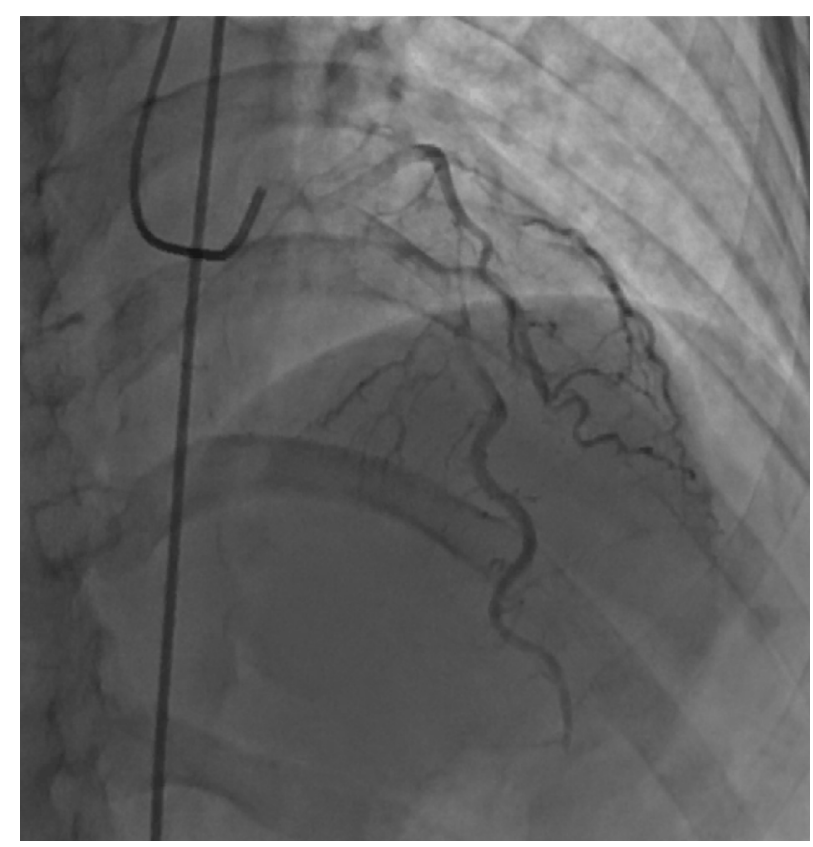

VIDEO 1. Coronary angiography demonstrating the distal occlusion of the left anterior descending coronary artery. Video available at: http:// www.jtcvsonline.org/article/S0022-5223(17)32380-2/fulltext.

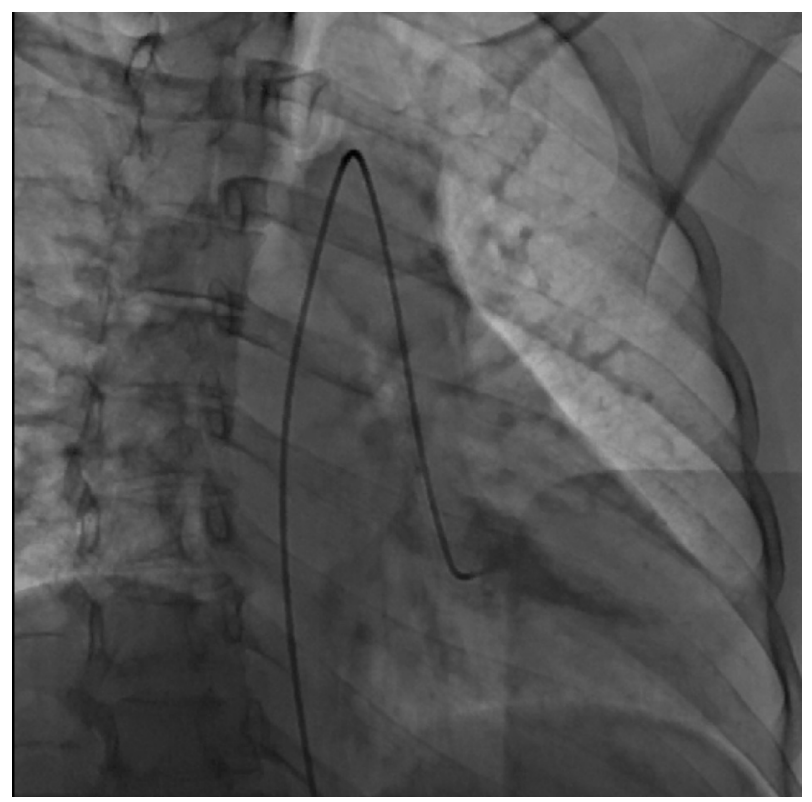

VIDEO 2. Ventriculography demonstrating the dilatation of the apex of the left ventricle and the akinesia of this region. Video available at: http://www.jtcvsonline.org/article/S0022-5223(17)32380-2/fulltext. 

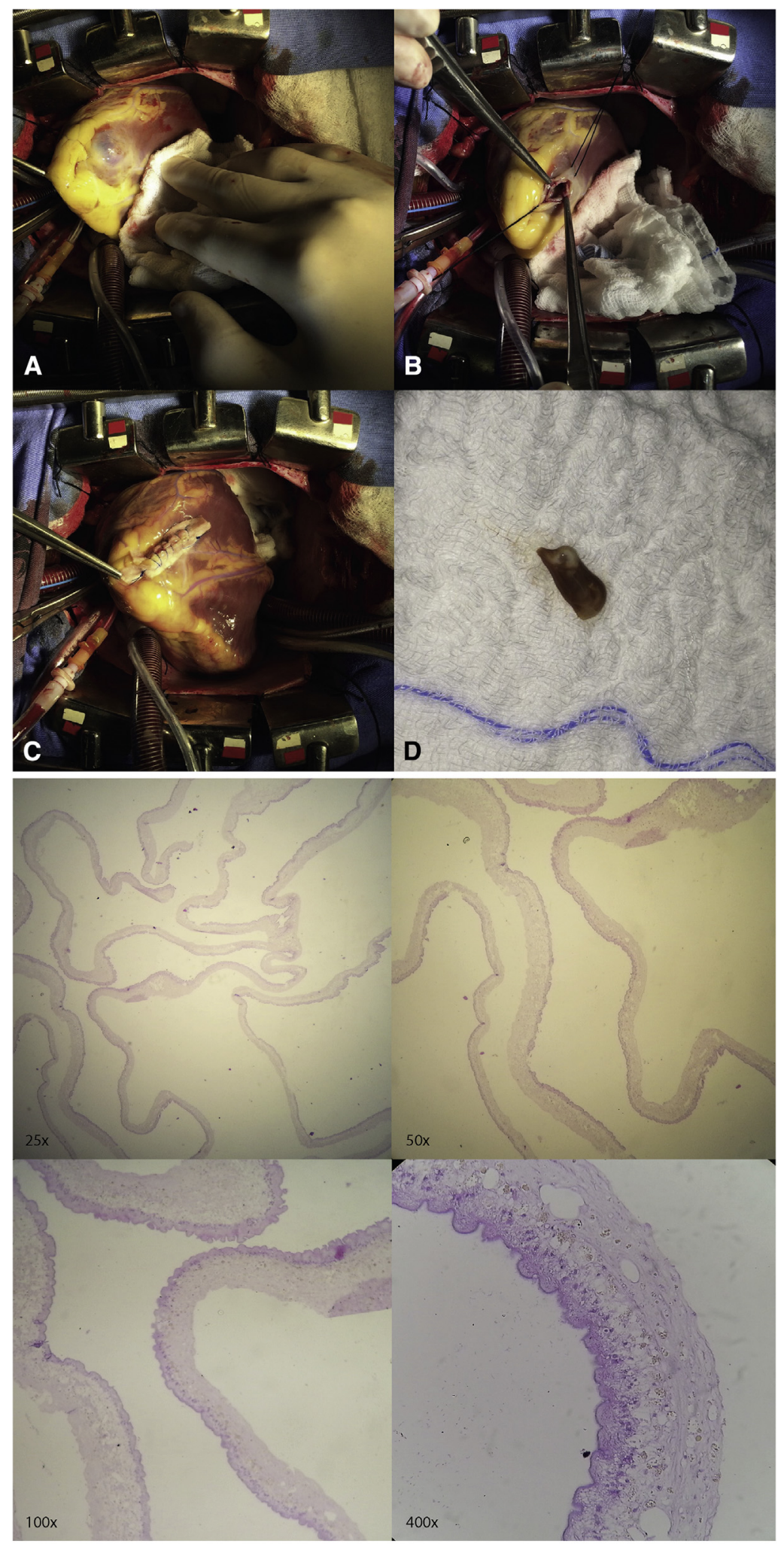

E

FIGURE 2. A, The cyst in the apex of the left ventricle, exerting external compression on the left anterior descending coronary artery. B, Interior view of the cyst. C, The aspect after the correction of the lesion as conventionally done for a ventricular aneurysm. D, The contents of the cyst. The content was a flat, elongated brown structure $15 \mathrm{~mm}$ in size; its integrity was preserved during the enucleation. E, Fragment of the wall of a cysticercus in the histologic study. 